\section{ESTRATEGIAS VERTICALES, DEMARCACIONES HORIZONTALES}

VERTICAL STRATEGIES, HORIZONTAL DEMARCATIONS

Iñigo García Odiaga; Iñaki Begiristain Mitxelena
1. Fotomontaje de un dirigible amarrado al

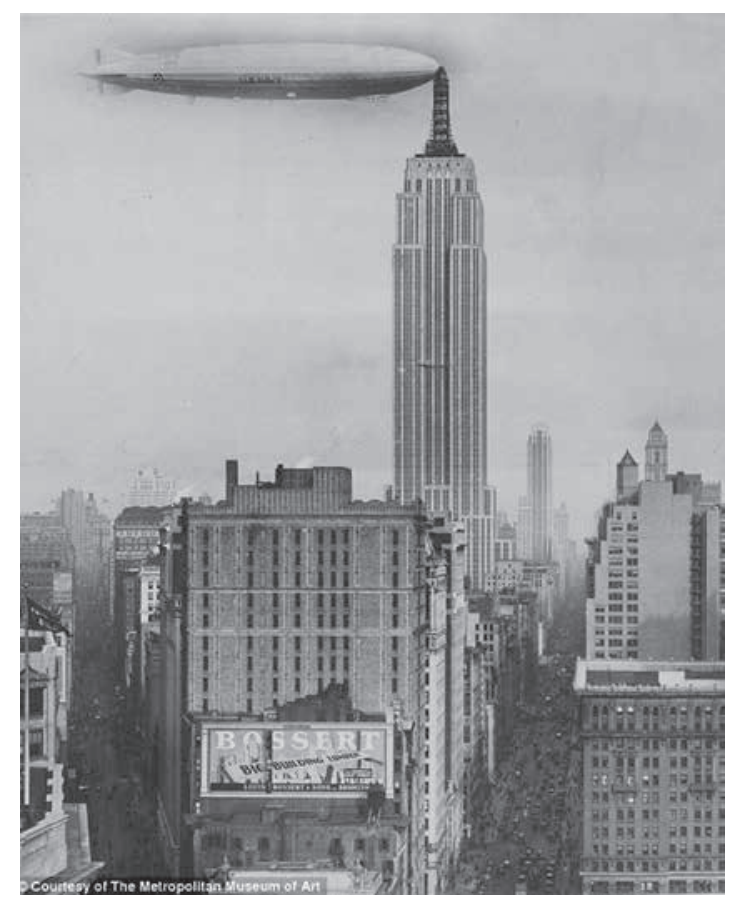

"E ntonces yo cogí unas planchas de acero muy SÍMBOLOS E HITOS DE REPRESENTACIÓN DEL E poderosas (..., más o menos la primera tenía LUGAR, Y POR CONSIGUIENTE DE LA COMUNIDAD Entonces hago una serie de formans quetros de espesor. Existen básicamente dos maneras de marcar un lugar: fí con esos problemas de los que he hablado, que pueden el campanario, el minarete, el menhir o el fuego ritual permente, de repente, noté como si fuera una corroboración potenciar eso, digamos, esa proyección del espacio y en el proceso de trabajo, en mi estudio, pues claro, yo esta pieza la veía vertical y un día la coloqué horizontalpor problemas de trabajo, y estando la pieza colocada horizontalde lo que yo había pensado, pero absoluta, es decir, que se producía al proyectarse el espacio a través de los huecos contra el suelo. Es como si la mesa, que yo llamo mesa, estuviera sostenida por esa proyección más que por las tres patas que yo le había puesto (....'m.

1. Transcripción de la conferencia impartida por Eduardo Chillida en la Universidad Internacional Menéndez Pelayo el 2 de agosto de 1985. Chillida, Eduardo:

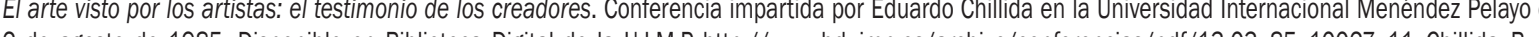
2 de abosto de 1985. Disponible en Biblioteca Digital de la U.I.M.P. http:///www.bduimp.es/archivo/conferencias/pdf/13-03_85_10027_11_Chillida_Bue

secerían al primer tipo (figura 1).

Serían ejemplos de lo segundo las murallas, tapias y cercas, en todas sus escalas, construcciones utilitarias y defensivos que demarcan un recinto y diferencian un dentro y un afuera. Su valor trasciende lo meramente utilitario y se convierte en representación de la comunidad que habita su interior.

Puede fácilmente elaborarse una secuencia de pares de conceptos opuestos en representación de cada stockades and walls were derived from defensive needs; the vertical elements, menhirs, obelisks and towers, from the desire for

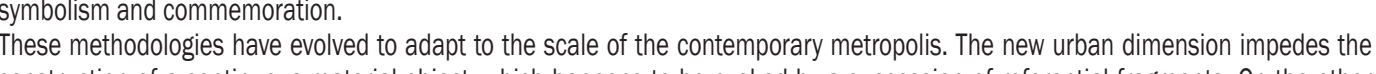
construction of a continuous material object, which happens to be evoked by a succession of referential fragments. On the other KEY WORDS horizontal skyscrapers; i Lissitzky; barriers; wall; door; intermodal.

Persona de contacto / Corresponding autor: i.garcia@vaumm.com. Arquitecto por la Escuela Técnica Superior de Arquitectura. Universidad del País Vasco 


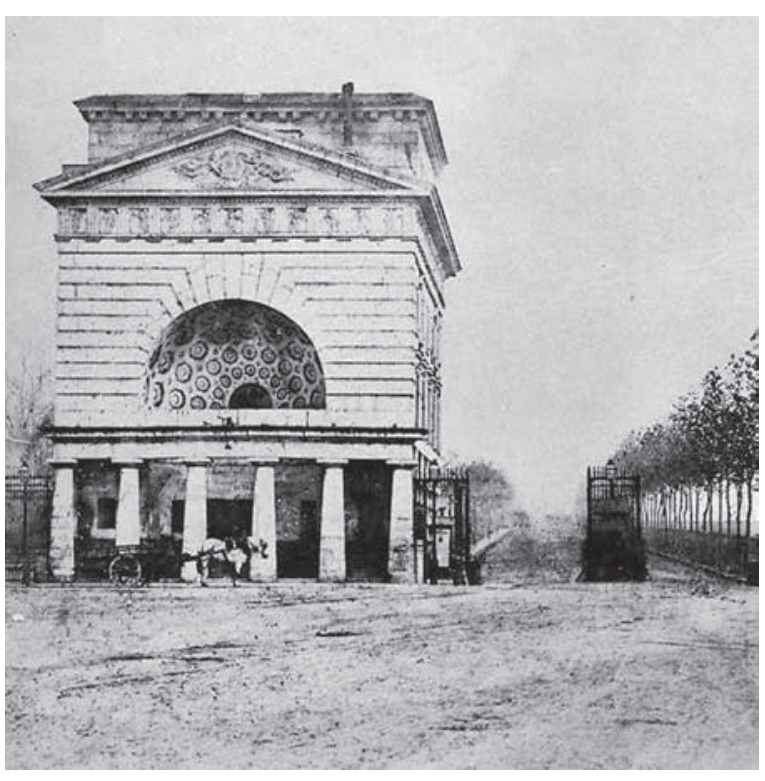

horizontal, Ileno/vacío, fálico/hueco, teocracia/democracia, monarcia/socia, fllich do coexisten y son complementarios.

LA PUERTA COMO EVOCACIÓN DEL LÍMITE

a función utilitaria del elemento "demarcador", constituyente del perímetro, requiere la preservación de su integridad física, la continuidad a lo largo de toda su longitud. Su función simbólica sin embargo puede construirse mediante la concurrencia de solo algunas de sus partes. E crómlechneolítico por ejemplo no constituye un borde con-

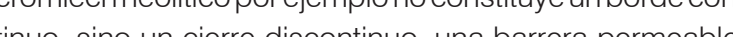
En su grado máximo de de

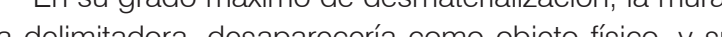
presencia sería evoceda por uno de ous paltesco, y su ventes más singures Lapresencia de la puesta evoca

En 1752, Marc-Antonie Laugier escribía la primera edición de Essai sur l'architecture en el que se lamenta de que los extranieros, al entrar en la ciudad de París encontraban difícil de creer que no estuviesen aún en alguna localidad cercana ya que nada representaba la entrada en la urbe del monarca francés En ese sentido, Laugier, propugnaba la construcción de unos grandes arcos del triunfo, que señalasen en todos los accesos radiales al centro urbano, ese hecho físico de la entrada que diesen presencia al lugar de paso a partir del cual uno se encontraba en París. Una solución similar a la que tenía Madrid con sus cinco puertas reales, Segovia, Atocha, Bilbao, Toledo y Alcalá, de las cuales, aunque con un proceso evolutivo en forma y ubicación, pueden aún contemplarse estas dos últimas. Estos accesos reales, además de simbolizar la entrada al espacio en el que residía el poder del rey, servían como lugar de registro y cobro de impuestos a todas las mercancías que entraban en la metrópoli.En 1782, el recaudador general de París, Lavoisier, propuso un nuevo recinto fiscal para la capital francesa, que pretendía la construcción física de nuevos accesos que ampliaban el ámbito de la muralla demolida en 1672 .

Cincuenta y cinco puntos de entrada, unidos por un boulevard circular, que circundaba el centro de la ciudad proyectadas por Claude-Nicolas Ledoux, comenzaron a levantarse en 1785. Para Ledoux, el encargo represento la cima de sus aspiraciones como arquitecto, por un lado suponla la mejora general de la penteria urbana y por otra, el embelecimiento monumental delos accesos cias le los a ne. Les de recaudación en pequenas of

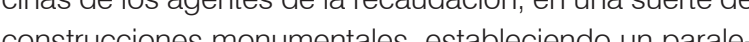
lismo con la peticín que años antes hab́a hecho Latgier Cono ́́t mismo reconoció, "de simples oficines de

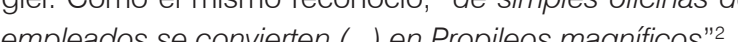
Ledoux propuso un coniunto de piezas, macadas

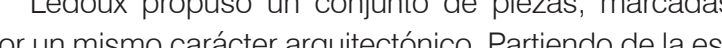
qurematzación de prototipos ćásicos o rencentistas y mediante procedimientos de combinacín y rentistas y ción iba conformando las diferentes unidades. Por este motivo, una tensión formal una especie de ley genética está presente en los diversos templos barrera, como hubiesen sido coados por la metamorfosis lenta de un único modelo original.

Esta condición estilística convierte las barreras, en un sistema global. En la contemplación de una de ellas están presentes todas las demás. La gramática transformacional que da lugar a cada una de estas piezas habí sido calculada para formar una cadena de asociación, capaz de estimular la percepción de los habitantes de la ciudad y tener de algún modo presente todo el sistema desde uno de sus elementos.

Puede afirmarse por tanto que se trata de un estilo, calculado a escala urbana, un estilo que más allá de de ender valores simbólicos, de severidad, de poder o de fuerza, para representar el recinto de una ciudad

EL APOYANUBES

Esa misma idea de sistema urbano, también subyace en el proyecto de los Apoyanubes que El Lissitzky ideó para Moscú. El documento fechado en 1924, enraíza en el espiritu de las vanguardias rusas, claramente vinculadas como movimiento artístico a los cambios sociales y a

dentidad que la revolución bolchevique pretendía sacar adelante (figura 3)

Durante la revolución el diseño gráfico o la pintura

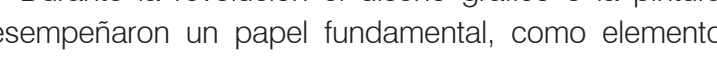
destacado para la divulgación del lenguaje político, hasta el extremo de que en 1917 se constituyó el ProletKult. "Cultura Proletaria" fue una institución que aspiraba a modificar radicalmente las formas artísticas existentes ediante la creación de una nueva y revolucionaria estética de la clase obrera, de modo que lograse transforma a Rusia agraria en una sociedad moderna industrial. En práctica, los artistas del ProletKult tenían como misión fun-

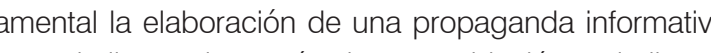
capaz de llegar al corazón de una población mal alimenEn con graves deficiencias de alfabetización

En este sentido es fácil comprobar cómo las ansias coctivas de una sociedad fueron capaces de representarse mediante una nueva manera de entender el arte, que cristalizó en las vanguardias rusas ${ }^{4}$.

Desde los primeros meses que siguieron a la Revolución de Octubre los artistas de vangurdia principalmente los futuristas, abrazan con entusiasmo la nueva causa politica. Unidos a ella por un mismo af́n de derribar un orden establecido que les negaba su reconocimiento se proclaman "depositarios del arte de la juventud", Ésta identificación es correspondida, al menos en un primer momento, también por los nuevos dirigentes. Malevich es nombrado profesor en la Academia de Bellas Artes, Chagall comisario de Bellas Artes y Kandinsky, además de profesor en Moscú, funcionario del gobierno encargado de fundar la Academia de Ciencias Artísticas.

Aunque el reconocimiento por parte de los líderes del cambio social nunca llega a ser completo y persisten algunas reservas que con el tiempo llevarán a la marginación oficial de la vanguardia artística, una parte de sus respectivos discursos es coincidente. Así las palabras de Lenin en su artículo La organización delpartido y la literatura del partido: "Nosotros, los socialistas, hemos de desenmascarar esa hipocresía y arrancar las falsas insignias,

, des moeurs et de la législation. Madrid: Ediciones AKAL, 1994, p. 16 3. Vilder, Anthony: Ledoux. Madrid: Ediciones AKAL, 1994, p. 115. . Ésta relación entre el arte, la a rquutectura y el pulso social en las Vanguardias está bien recogido en: Frampton, Kenneth: Historia critica de la Arquitectur 
5. Dibujos de la propuesta de Wolkenbügel desarriolada por MartStam y Emil Roth

representan-
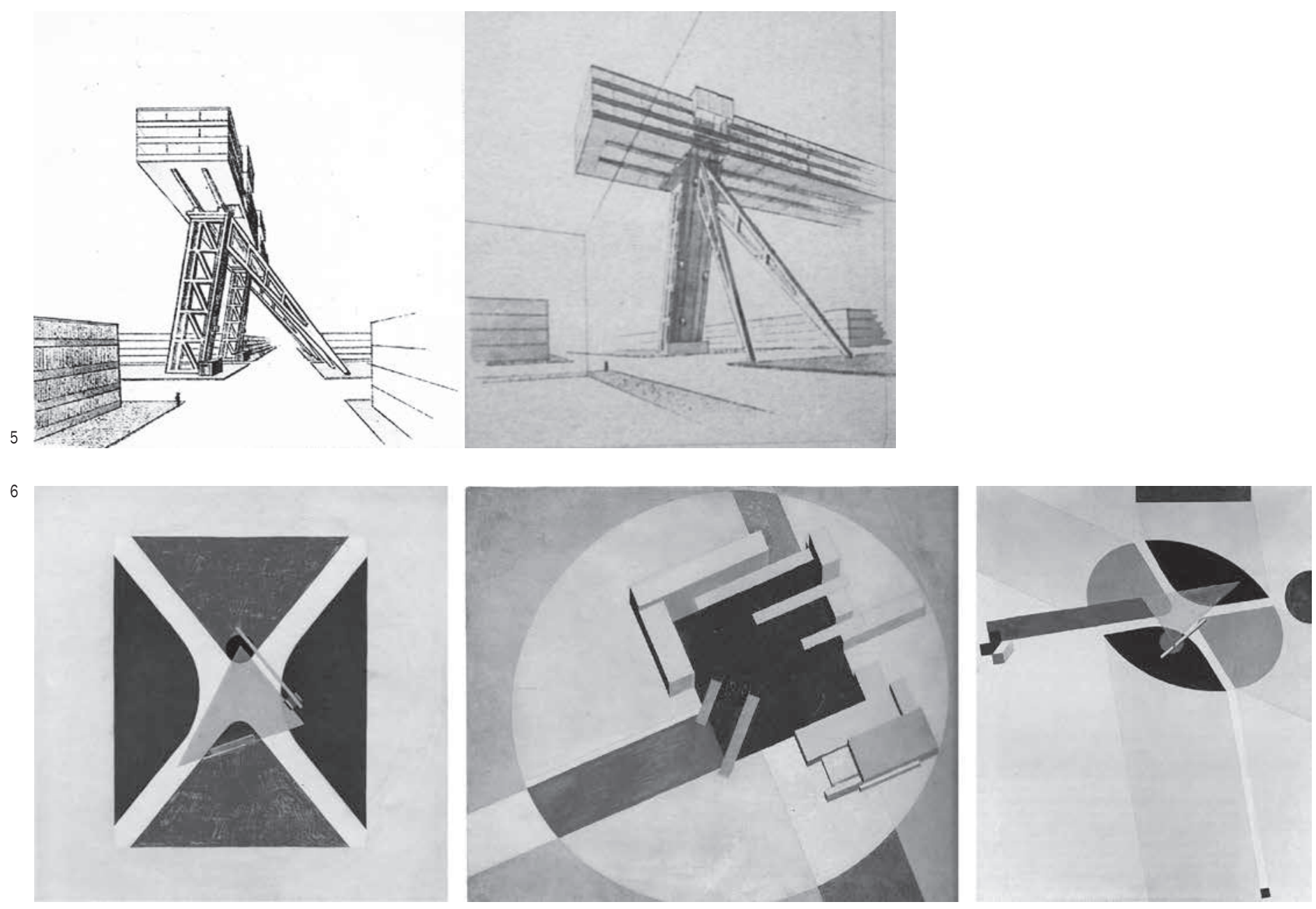

sobre soportes metálicos, con el fin de evitar las vibraciones producidas por el tráfico. Se subiría a ellos por medio de ascensores, y los niveles inferiores se reservarían para usos publicos, las viviendas se situarían en las plantas más allas y los al tráfeco discuriría bajo los edificios. En convalecencia por tuberculosis en Suiza, mantuvo El Lissitzky con Emil Roth. Roth aquejado de una enfermedad pulmonar similar a la del propio El Lissitzky, había tenido (ave abandonar sus estudios en la ETH de Zürich, donde estaba cursando cálculo estructural con Robert Maillart. Mart Stam que el 9 de febrero de 1924 recibí́ al El Lis-

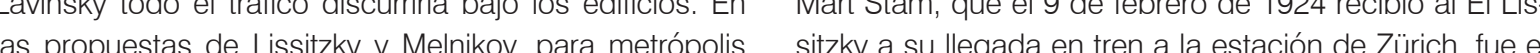
ya ponstruidas, a "capa superior" se resena para nodos que les sugirió que podrín colaborar para desar fue $e$ estratégicos?

Todo este desarrollo "creíble" del sistema estructura proyecto de los Wolkenbügel. Roth estudió varias alterdel proyecto, se debe a la colaboración que durante su absorber deformaciones asumibles.
Parece que además Roth, aportó al edificio condicionantes materiales y formales importantes. Aconsej pasar del hormigón armado ideado por El Lissitzky, a una solución de acero más ligera y constructivamente más controlable. Además parece que fue fundamental ta mentros flecto de sus solicitaciones estructurates. Pero desde una óptica formal la solución aporta al coniunto una mayo ingravidezy ligeza ayudando a la visión del proyecto como de una gran máquina a unto de ech a avolar (figura 5). Posteriormente Emil Roth expreś sus dudas sobre istem estucturat enpleado y unto a Mer Stam, proyectó un nuevo modeb de edicio Apoyanubes. Este verzo, de idea de la importancia que Stam. Rothy otros muy cercanos a El Lissitzy que junto áthab́n formado el grupo de redacción de la "ABC Beitrage zum Baven", otorgaban al proyecto y a sus valores, sociles, tecnob́gicosy revolucionarios La propuesta de Emil Rothy Mert Stam, muestra un cuerpo horizontal de tres plantas que se apoya sobre una estructura con forma de trípode. Las tres patas que configuran el apoyo, arrancan inclinadas, una de ellas alberga las escaleras y ascensores, mientras que las otras dos de sección triangular muestran a las claras su función propiamente estructural. En su coniunto la estructura podría calificarse de menos depurada ya que está centrada en expresar a las claras la capacida estructural del sistema de apoyo.

Los Wolkenbúgel tenían su antecedente dentro de la obra de Lissitzky en las pinturas y dibujos de la sería de vogo (Proyecto para la afirmación de lo nuevo) 9 . Dichos trabajos, que Lissitzky definía como trabajos de transición entre la pintura y la arquitectura, entres las dos y las tres su aponacion para que elcuerpo superior del edificio, se

dimensiones, representaban construcciones utópicas suspendidas sobre un sustrato en el que se invitaba a ver el universo (figura 6).

s representaban esferas flotantes, que inviaban al espectador a hacerlas girar mentalmente. Seautor "cuando giramos el lienzo, tenemos sensación de entrar en el espacio y hacernos parte de la

Como objetos, como máquinas, los Wolkenbüge on con lugar concreto en cacion, tuvieran una importan estuvier supeditados a determinacion y por conte perior. En el plano de 1926, las ocho piezas, aparecen radiante orientadas hacia el Kremlin mostando que entre ellas existe por tanto una estategir gen a que las ordena, una ley que pretende acturer en la gran escala del paisaje urbano de Moscú Las ocho torres se iban a ubicar en los cruces, en los nodos, formados por la intersección de las avenidas radias que parten del Kremlin al encontrarse con el primer anillo de circunvelación de la ciudad el conformado por los bulevares Tverskoy Miasnitzkaya o Petrosky

Este primer anillo, formado por anchos bulevares ajardinados, que circunda el centro de Moscú, es el resultado del derribo de la muralla de la ciudad en el siglo XVII, por lo que es difícil abstraerse ahora del valor meta fórico del proyecto de El Lissitzky como rememoración de las puertas de la antigua Moscú. Un significado que Kenneth Frampton les otorga al calificarlos de propileos elevados

Un año antes de la publicación de la propuesta de Lissitzky, en 1923 se había presentado el plan para el Nuevo Moscú, dirigido por el ex-académico Alexei Schusev desde posiciones alejadas de la vanguardia. Lissitzky explicaba en la presentación del proyecto, que su intención

7. Kahn-Magomedov, Selim O. PPioneers of Sovvet Architecture. Londres: Thames and Hudson, 1983, pp. 279-280,

8. Burgos, Francisco; Garrido, Gines: El Lissitzky. Wolkenbügel 1924-1925. Madrid: Editorial Rueda SL, 2004, pp. 56-57.

9. The Solomon Guggenheim Foundation: The Great Utopia. The Russian and Soviet Avant-Garde, 1915-1932. New York: The Solomon Guggenheim Foundation 1992, pp. $125-126$.

10. Tsanisanogolou, María: La síntesis de arte y arquitectura en la vanguardia rusa. El testimonio de la colección Costakis. Barcelona: Fundación La Caixa

11. Frampton, Kenneth.H Historia Critica de la Arquitectura Moderna. Barcelona: Editorial Gustavo Gili, 1980, p.169-174. 
7. Plano de Moscú con las ubicaciones de los ocho
Apoyanubes, publicado por El Lissitzky en la revista Apoyanubes
ASNOVA

8. Mesa de Omar Khayyam II, Eduardo Chillida.
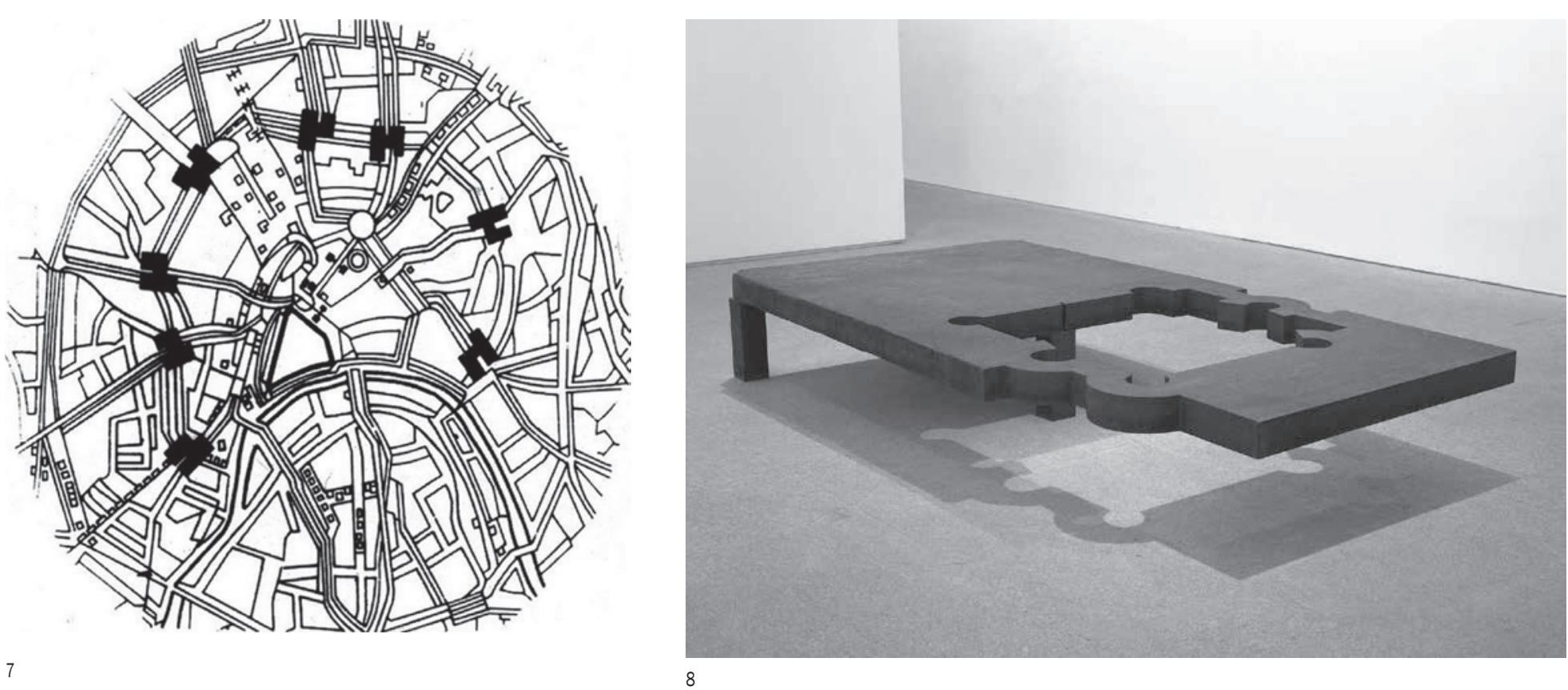

era crear oficinas adecuadas para las nuevas exigencias, adaptándose al sistema urbano centralizado del viejo Moscú. Son al igual que las puertas de la ciudad antigua elemento de referencia urbana, hitos o indicadores dentro de la trama de la ciudad (figura 7). Tal y como el propio Lissitzky argumenta en el artículo de ASNOVA, uno de los objetivos del proyecto era: "aportar una nueva escala a la ciudad en la que el hombre de hoy en día nunca más mida su entorno con el auxilio de sus propios codos, sino con centenares de metros"12.

En este sentido, desde el punto de vista del paisaje urbano, los Wolkenbügel, se presentan como hitos del skyline urbano de la ciudad, que ayudan a la orientación de sus habitantes. En la publicación original de proyecto en el numero 1 de la revista ASNOVA en 1926 El Lissitzky incluyó seis esquemas que a modo de sus composiciones PROUN, representaban visiones del los Wolkenbugel. Visiones intermedias, entre alzados, persy el volumen de los edificios, vistos desde arriba, abajo

hacia el Kremlin, desde el Kremlin, a lo largo del bulevar o desde el lado opuesto. Mediante estas representaciones Lissizky olorga a los edificios el valor de un sistema de señales, el valor de un sistema de códigos capaz de guiar a los habitantes de Moscú, por el paisaje urbano de paciudad, como silos edificios se transtormasen en faros para los peatones.

Como el autor narra en el texto que acompaña las imágenes publicadas "Esta caracteristica sive para faciItar enormemente la orientación dentro de la ciudad [... Elmontaje de toda la serie y la introducción delcolor para Ientificar cada uno de los rascacielos de forma inaividuaIzada, sevirá para apuntalar sus favorables cualidades de cara a la orientación"

Aunque no hay documentación que pueda explicar cómo estaría pintada cada una de las ocho torres, sí que es color como elemento distintivo de cada una de las construc-

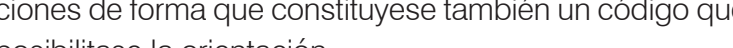
posibilitase la orientación.
HORIZONTAL FRENTE VERTICAL

Pero también es innegable el valor de la propuesta de $\mathrm{E}$ Lissitzky, como construcción de un espacio urbano. Algunavez se ha definido la arquiltectura Moderna, comola primera en permitir a la gente, no solo rodear el edificio, cruzarlo, o estar dentro, simo tanibién pasar por debajo de é. El Apoyanubes se eleva tambien para generar un espacio urbano sorprendente, caracterizado por el vacio que queda debajo, tan importante en el proyecto como la gran estructura superior. Los peatones y el tráfico denso que recorre la primera circunvalación del centro, tiene una visión fugaz de la pieza, inestable. Una visión que $\mathrm{E}$ Lissitzky plasmó en diferentes fotomontajes, en los que nunca podian verse de forma simultanea las tres patas aumentando aún más el desapego de la estructura por el suelo, y multiplicando si cabe el reto de enfrentarse a gravedad desde el punto de vista de la percepción. fuerzas y tensiones, si se quiere sensaciones, que transorman el espacio de la plaza en monumental.

Es tanto una consecuencia, como una formulación de a idea de ingravidez, del desafio que supone el volar do la estructura, que la pieza, casi una escultura, concita. Es fácil ligar esta reflexión con aquella que Eduardo Chililia relataba y ponía en relación con su trabajo, muchos años más tarde, con las mesas Omar Khayyam. "Un dia estaba yo, despues de estar trabajando en Legazpl, en una tabrica, en Patricio Echevarria, estaba cansado y sentado en una nave grande en el suelo, fumando y esperando que esluvese preparada una cosa que tenia que hacer en otro sentido completamente distinto. Y estaba en esta siluacion relajada en que se suele le yo no estaba pensando en nada, y vi en el fondo de la neve, una nave enome, una serie de operaciones que séclaban realzando con objelo de levantar una gran máquina muy pesada, que estaba en el fondo, alli, la esy llevarla a otro sitio Yo, mientros estaban haciendo y lievarla a orro silio. Yo, mientras estaban haciendo esta quinal y me daba la impresín de que todo el espacio
Es por este motivo, por el que el vacío inferior, concita estar cuando uno no está haciendo nada, verdaderamen-

que habia encima estaba aplastando la máquina contra elsuelo. Yo notaba esa sensación de gravitación del espacio contra la máquina. Y en elmomento en que de esa un . todo el espacio de la fabrica se metia debajo y ayudaba antar la máquina

Aligual que en los folomonlajes de El Lissitzky, las mesas de Omar Khayyam aparecen casi siempre fotografladas ocultando alguna de sus patas. Sus proporciones dificultan voluntariamente la visión simullannea de las tres patas, de manera que reproduce en el espectador la impresión que tuvo Eduardo Chillida en aquel momento de "revelación" (figura 8).

Este recurso del desario a la gravedad tiene sin duda en el caso de EI Lissitzky el valor simbolico anadido de epresentar a la revolución como oposición a un poder absoluto, pero la fascinación por el desafí estructura que supone el voladizo, es una constante en la vanguardia arquitectónica del siglo XX. El desaflo a los Iimites de la resistencia de los materiales como simbolo de audaci se sigue repitiendo una y otra vez.

Ejemplos como el Banco de Georgia de Georgly Chakhava, o The Interlace City, proyectado por OMA para Singapur, representan versiones contemporáneas de ese mismo rascacielos horizontal. Aquil, en oposición a quienes disputan la carrera por construir el edificio más alto, la importancia no recae tanto en las dimensiones como en la proporción

Probablemente algunos de los ejemplos que más puedan recordar al proyecto de El Lissitzky son los de Ste ven Holl para el museo de Nanjing y para el Vanke Center en she ent y sobre todo sus estudios para el lintite urbano de Phonix. Estos ultinos comparten cón el proEl proyecto de 1989 desarrollado por Holl ner to dad de Phoenix, recibió el sobrenombre dBarras de

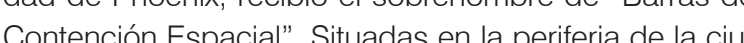
dad esta serie de piezas mar a límite de la ciudad

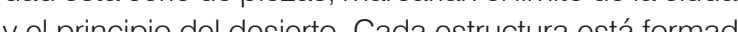
ligual que las de El Lissitzy tanto por una estructura

14. Transcripción de la conferencia impartida por Eduardo Chililida en la Universidad Internacional Menéndez Pelayv el 2 de agosto de 1985.

12. Platonov, Andrei: Chevengur. Michigan: Ed. Ardis, 1978, p. 288.

13. Ladowsky, Nicolai A. : Lissitzky, Lázar M. : ASNOVA n¹. Moscu: Asociación de Nuevos Arquitectos (ASNOVA, 1926, pp. 2-3. 
alámbrica, como por un gran vacío, capaz de enmarcar las montañas lejanas y el desierto, conformando un nuevo horizonte, una nueva relación entre la ciudad y e

A pesar de la fuerte abstracción de las formas seriadas propuestas por Steven Holl, que son fácilmente equiparables a las variaciones de cubos abiertos incompletos de Sol LeWitt, éstas no buscan desvincularse por completo del contexto, sino que incorporan mecanismos para eivindicar su pertenencia al lugar, e incluso la memoria

Las secciones constructivas de los brazos, están resueltas con una dimensión de $9 \times 9$ metros, una medida que entra enr dación con el orro gran elemento construio metros de ancho, construidos por los ind los Hohy antiguos monach mismo modo la materialidad de ba barras de contencín mispacil busca también fijarse a lugar Por esa racón el hormigón armado que formá a lu prras esa razón, e rí con la utilización de áridos provenientes del propio desieto. En cierto modo, esas estructuras espaciales abiertas, podrín entenderse como el resultado de Iormas, pó́trica a la materia del desierto, en su contacto con el límite urbano.

EL TRANSPORTE COLECTIVO COMO AGLUTINADOR DE LA METRÓPOL cala urbana tiene otro efecto, digno de mención el de evidenciar el de poner de manifiesto los nudos de la red evidenciar, el de poner

En estos puntos clave de la ciudad, el Apoyanubes, convierte también en un nodo de transporte, ya que EI Lissitzky contemplaba que una de las patas albergara una parada de la red de metro de la ciudad y las otras dos, sendas paradas de tranvía. Una decisión que llamativamente 10 años después, cuando comenzaron las obras del metro de Moscú, se vio ratificada ya que todos esos puntos singulares de la ciudad cuentan hoy con importantes intercambiadores de metro.

En la dialéctica de pares opuestos propuesta al inicio de éste escrito, surgen a veces ambigüedades que dependen de la escala de lectura. Tomemos por el Empire State. Cuando se construyó fue el edificio más

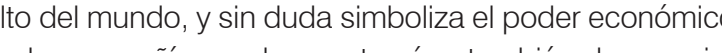
de una ciudad y pals. En ese sentido pertenecería al primero de

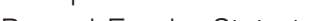

Pestaba previsto como diseñada con ése propósito. Aunqu postorioción fué comprobó que las condiciones de viento no permitín l cesembaco de pasajes y únicamente un pequeño diricible llegó a amarrarse al edificio durante unos pocos minutos, diversos fotomontajes de la época represenaron la imagen del edificio como pueto de amarre. Es una imagen polersa que ce superpon a lactura de edificio como torre de refencia y que orece una lectula más sutil colocando al Empire State, como parte de una extensared de puntos de atraque, como nodo de un sistema mayor. Del mismo modo aue las campanas de varies iglesis sonando a unisono ponen de manifiesto la existencia de un suprasistema que abarca un ámbito superior y más extenso, al que pertenece la torre de iglesia local el dirigible amarrado a la cumbre del Empire State, matiza la singularidad del gran edificio y anuncire existencia de más torres en otros lugares. Como probablemente no podí ser de otro modo esta matización se realiza contraponiendo a la vertical absoluta un elemento erfectamente horizontal.

Aparece así otra manera de representar la colectividad, que se complementa con las dos anteriores, y cuya lectura puede superponerse a ellas. A estos mecanismos, centrados en la elevación vertical de un punto, la demarcación de un recinto, se les añadiría la representación de una red mediante la materialización de sus nodos

El gran atractivo de la visión de El Lissitzky consiste así en identificar un nuevo modo de demarcación de la ciudad, a la escala de la metrópoli moderna. Es una estrategia que se apoya en el recurso intemporal de la muralla, de la que toma su trazado, y en la referencia a las puertas de la ciudad, que formalmente simboliza mediante la construcción de un dintel gigantesco, pero que va más allá. La entidad unitaria de la metrópoli ya no se representa por medio de sus límites exteriores, sino a traves de las redes de transporte que la estructuran.
Estas redes no se manifiestan con demasiada nitidez, no tienen una gran presencia física en el paisaje urbano. Su materialidad no tiene nada que ver con la de las aniguas calzadas o acueductos. Son casi invisibles, como la linea del tranvia y la red de metro sublenáneo. Aunque mucho más livina y ye manifiestan mediante la consmuchón ás livana, y se manifestan mediante la cons-

Los nuevos elementos de demarcación no repreanta que se irá desarrollando en el tiempo. La estacín inque se iá desarcambiador de transporte se convierte en la representación de la nueva estructura del sistema metropolitano

a identificación de esta nueva estructura no depende ya de la visión inmediata de su continuidad, por ello el tamaño de sus componentes deja de tener importancia. El proyecto de El Lissitzky conserva aún la monumentalidad de la puerta de la ciudad, pero anuncia la vía para otro tipo de presencia, que se hace visible no ya por e ese sentido quizás deberíamos buscar sus equivalentes en las bido quizás deberlamos buscar sus equivalentes són contemporánea creada por Norman Foster para metro de Bilbao. Elementos que prácticamente podríamos calificar de "mobiliario urbano", si los analizásemos individualmente. Un ejercicio éste, imposible, ya que como en las barrières de Ledoux, una boca de metro nos conecta, y no solo físicamente, sino metafóricamente, con todas las demás. Mostrando a los habitantes de un determinado lugar, su pertenencia a una colectividad, a un grupo de ciudadanos con intereses similares y fijados a un ámbito territorial por su capacidad de desplazarse por una red.

Es esta lectura de el proyecto de El Lissitzky, la de sistema urbano, la que desde la contemporaneidad más réditos puede aportar. Un buen ejemplo puede ser el de a ciudad colombiana de Medellín. Hasta hace pocos años la ciudad lideraba el ranking de las ciudades más

源

16. http://www.plataformaarquitectura.cl/2012/02/03/cai-periferico-medellin-edu/ violentas de Latinoamerica, y su imagen se asociaba a una periferia caótica, sin presencia del estado y sin ninguna cohesión socia

Uno de las primeras intervenciones puestas en marcha para la regeneración de la ciudad fue el llamado Metrocable, un teleférico que hace las veces de metro urbano sobrevolando los tejados de la escarpada topografla del borde norte de la ciudad. Al igual que en los Wolkenbugel, sus estaciones incorporan pequenos espacios públicos, equipamientos comuntianios, guardeligs, biblotecas o centros sanilanios. Esta intenvencion más al transporte público y que se aleja del urbanismo

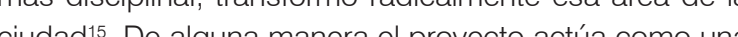
cocupunt De alona capaces de transformar un lugar residual del extrarradio, en un nodo más del sistema urbano y por ende inclio en la ciudad El estado, la administración resurge as' berrios olvidados, generando una nueva actitud en los vecinos, a modo de revolución social se siente de nuevo parte de la colectividad.

Otro proyecto desarrollado en Medellín, en la mis- intervención y con grandes similitudes

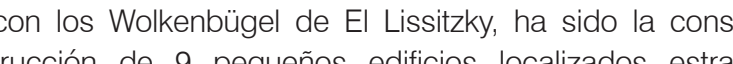
tégicamente en zonas de la periferia de la ciudad Ubicados en lo alto de las laderas, se erigen como nodos de un sistema mayor allí donde el caos urbano de la favela era el protagonista. Estos edificios se convierten en referentes urbanos dentro de su contexto y se levantan como símbolos de la comunidad y del bario para insertarse en la red de la ciudad.

La arquitectura de estos modestos edificios, está desarrollada para centrar su esfuerzo en ser reconocibles en la distancia. Su forma de torreón, los convierte en hitos que sobresalen en el paisaje. Al iqual que los rascacielos horizontales de Moscú, cada uno de ellos está caracterizado por un color, dejando claro que a pesar de poseer una misma genética que los une, son piezas independientes. Los nueve edificios, por su estratégica 


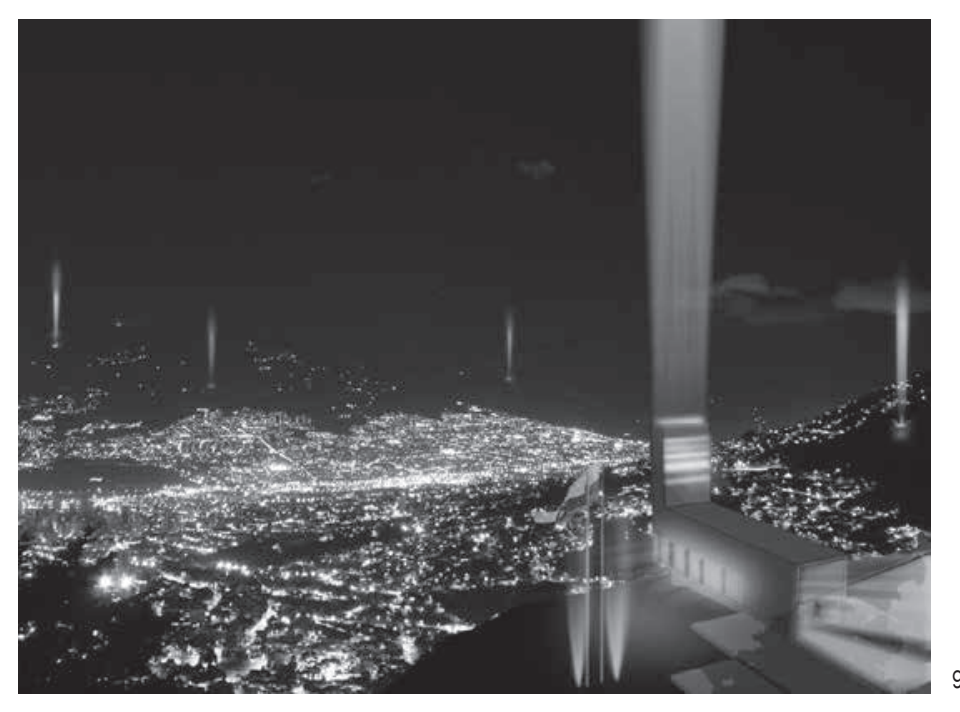

hermana Sophie desde su retiro en Minusio en las laderas de Medellín a través de la luz (figura 9). en 1925

A modo de faros nocturnos, sus proyectores de colores "Sipuedes, no te olvides de conseguir un plano de Mosiluminan el skyline de la ciudad, introduciendo en la car- cú. Cada vez estoy más convencido de que mi obra tiene lografila urbana barrios a los que antes lo colectivo nunca mucho que ver con los problemas de nuestro tiempo. Se lehabía llegado. Más alla de su valor como arquitecturas, vanta sobre tres apoyos y cuelga. ¿No es una locura? Ni los podemos calificar a estos edificios de enlaces entre los franceses, ni los holandeses (los alemanes un poco más) Estas intervenciones arquitectónicas, que partiendo desde una escala casi puntual, llegan a adquirir una escala de infraestrucural, se enfirnta a visiones que dirigen el urbanismo a intervenciones de gran escala. Proyectos pueden entender nuestra voluntad arquitectónica, pero perciben su fuerza. Suincomprensión refuerza mi convicción" La ciudad, lo colectivo, no llega ya hasta los limites de a muralla, ni comienza en la puerta de entrada. La metrópoli alcanza hasta donde to hacen sus redes de transporte rizado un sentimiento de pertenencia a la red urbana.

\section{Bibliografía}

Burgos, Francisco; Garrido, Gines: El Lissitzky. Wolkenbügel 1924-1925. Madrid: Editorial Rueda SL, 2004.

Chillida, Eduardo: El arte visto por los artistas: el testimonio de los creadores. Conferencia impartida por Eduardo Chillida en la Universidad Intern 03 85 10027 11 chillid 2 de agosto de 1985 Dist

De Feo, Vittorio: La arcutectura en G URSS: 1917-1936. Madrid: Alianza Editorial 1979.

Frampton, Kenneth: Historia Critica de la Arquitectura Moderna. Barcelona: Editorial Gustavo Gili, 1980.

http://www.plataformaarquitectura.cl/2012/02/03/cai-periferico-medellin-edu/

http://www.platatorormaarquitectura.cl/2012/02/03/cai-perfierico-medellin-edu/

Kahn-Magomedov, Selim O. Pioneers of Soviet Architecture. Londres: Thames and Hudson, 1983.

Kahn-Magomedov, Selim 0.: : Pioneers of Soviet Architecture. Londres: Thames and Hudson, 1983.

Ledoux, Claude-Nicolas: Architectureconsideréesous le rapport de lấrt, des moeurs et de la législation, Madrid: Ediciones AKAL, 1994. PMCid:P

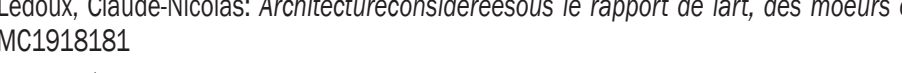

Lenin, Vládimirllich: Obras completas, Tomo X. Buenos Aires: Editorial Cartago, 1960.

Lissitzky-Küpers, Sophie: El Lissitzky. Life, Letters, Texts. New York: Thames \& Hudson, 1992.

Platonov, Andrei: Chevengur. Michigan: Ed. Ardis, 1978

The Solomon Guggenheim Foundation: The Great Utopia. The Russian and Soviet Avant-Garde, 1915-1932. New York: The Solomon Guggenhein

Isanisanoglou, María: La sintesis de arte y arquitectura en la vanguardia rusa. El testimonio de la colección Costakis. Barcelona: Fundación La Caixa/

Vilder Anthony: Ledoux. Madrid: Ediciones AKAL, 1994.
Inigo Garcia Odiaga (Bibao, 1977). Estudio en la Escuela Tecnica Superior de Arquitectura de San Sebastian, obteniendo el titulo de arquitecto en el año 2003. Master en Ordenación y Gestión del Territorio por la UPV en el año 2006. En el año 2005 publica el libro Florencio Mocoroa, arquitecto inaugurandol la colecclión del COAW Gipuzzoa. Fundo VAUMM arquntectos en el año 2003, ha sido seleccionado para los premios Mres Van der Rohe en 2013y finalista a los premilos COAW por el edinclo del Basque Culinary Center. haki Begiristain Mitxelena (Donostia, 1964). Estudilo en las Escuelas de Arquitectura de la Universidadd de Navarra en Pamplona CHU, en 2011 Ejere coms profesor 


\section{Autor imagen y fuente bibliográfica de procedencia}

Información facilitada por los autores de los artículos: página 17, 1 y 2 (Loghem, J. B. van: Bouwen / Bauen / Bâtir / Building Holland. Amsterdam: Kosmos 1932); página 18, 3 (Wiebenga archive (69-70), NAi, Rotterdam); página 20, 4 (Jan Molema), página 21, 5 (http://www.spotzi.com); página 22, 6 (Loghem, J. B. van: Bouwen / Bauen / Bâtir / Building Holland. Amsterdam: Kosmos 1932; Schütte-Lyhotski Archiv, Universität für angewannte Kunst, Vienna over a cadastral drawing on the internet: http://nah.cuzk.cz. Composed by Peter Bak and Jan Molema); página 23, 7 (Loghem, J. B. van: Bouwen / Bauen / Bâtir / Building Holland. Amsterdam: Kosmos 1932); página 24, 8 (Private collection Jan Molema), 9 (Jan Molema), 10 (Duiker Archive, NAi, Rotterdam); página 25, 11 (Van Loghem Bouwen / Bauen / Bâtir / Building Holland. Amsterdam: Kosmos 1932; C.A. Alberts and E.J. Jelles, Duiker 1890-1935, Forum, Amsterdam 1972); página 26, 12 ( Photo by courtesy of Arie den Dikken), 13 (Private collection Jan Molema); página 27, 14 ( Loghem, J. B. van: Bouwen / Bauen / Bâtir / Building Holland. Amsterdam: Kosmos 1932); página 28, 15 (Section from drawing in Duiker Archive, NAi Rotterdam); página 30, 16 (Multi-layer drawing on the basis of Duiker's site plan in Van Loghem Bouwen / Bauen / Bâtir / Building Holland. Amsterdam: Kosmos 1932. Composed by Jan Molema and Peter Bak); página 35, 1 y 2 (Francisco González de Canales); página 38, 3 y 4; página 40, 5; página 41, 6 (izda) (Koolhaas, Rem y Mau, Bruce: S, M, L, XL, Nueva York: The Monacelli Press, 1994), 6 (drcha) (Francisco González de Canales); página 41, 7; página 43, 8 y 9; página 44, 10 y 11; (Koolhaas, Rem y Mau, Bruce: S, M, L, XL, Nueva York: The Monacelli Press, 1994); página 49, 1 (Le Corbusier. En Boesiger, Willy. (Ed.): Le Corbusier Oeuvre complète. Volumen 1. 1910-29. 15a ed. Basilea: Birkhäuser Publishers - París: Fondation Le Corbusier, 1999. p. 189) ; página 51, 2 (Le Corbusier: Dibujo original del autor. FLC 10910. Fundación Le Corbusier. París, 1931), 3 (Le Corbusier: Fotografía del archivo de la FLC. FLC L2-4-41. Fundación Le Corbusier. París, s/f.); ; página 52, 4 (Le Corbusier. En Boesiger, Willy. (Ed.): Le Corbusier Oeuvre complète. Volumen 4. 1938-4. $11^{\mathrm{a}}$ ed. Basilea: Birkhäuser Publishers - París: Fondation Le Corbusier, 1999. p. 139) ; página 53, 5 (Le Corbusier: Dibujo original del autor. FLC 19238. Fundación Le Corbusier. París, 1936); página 54, 6 (Le Corbusier. EnBoesiger, Willy. (Ed.): Le Corbusier Oeuvre complète. Volumen 5. 1946-52. 11ª ed. Basilea: Birkhäuser Publishers París: Fondation Le Corbusier, 1999. p. 37) ; página 55, 7 (Le Corbusier: Dibujo original del autor. FLC 32294. Fundación Le Corbusier. París, 1951), 8 (Le Corbusier: Dibujo original del autor. FLC 2892. Fundación Le Corbusier. París, 1951), 9 (Le Corbusier. En Boesiger, Willy. (Ed.): Le Corbusier Oeuvre complète. Volumen 5. 1946-52. 11ª ed. Basilea: Birkhäuser Publishers - París: Fondation Le Corbusier, 1999. p. 121) ; página 56, 10 (Le Corbusier. En Petit, Jean. (Ed.): Un couvent de Le Corbusier. París: Éditions de Minuit, 1961. p. 112); página 58, 11 (Le Corbusier. EnBoesiger, Willy. (Ed.): Le Corbusier Oeuvre complète. Volumen 7. 1957-65. $7^{a}$ ed. Basilea: Birkhäuser Publishers - París: Fondation Le Corbusier, 1999. p. 33), 12 (Le Corbusier: Dibujo original del autor. FLC 31197. Fundación Le Corbusier. París, 1960), 13 (Le Corbusier: Dibujo original del autor. FLC 11644. Fundación Le Corbusier. París, 1963); página 60, 14 (Le Corbusier: Dibujo original del autor. FLC 28450. Fundación Le Corbusier. París, 1963), 15 (Le Corbusier: Dibujo original del autor. FLC 28460. Fundación Le Corbusier. París, 1963), 16 (Le Corbusier: A propósito del urbanismo. Barcelona: Editorial Poseidón, 1980. p. 144); página 63, 1 (From the archive of The Metropolitan Museum of Art, New York); página 64,2 (Courtesy of the digital archive of architectural images archINFORM), 3 y página 66, 4 (Wolfe, Ross: The Charnel-House. [en línea] New York. Disponible en www.thecharnelhouse.org); página 68, 5 (From the archive of the DEUTSCHES ARCHITEKTURMUSEUM), 6 (composed image, using different PROUNS of Lissintzky from www.wikipaintings.org); página 70, 7 (Composed image, using two drawing from the digital archive of architectural images archINFORM), 8 (From the permanent Collection of the Museo Nacional Centro de Arte Reina Sofía); página 74, 9 (Render of the project CAl Periféricos of the Empresa de Desarrollo Urbano de Medellín, EDU); página 78, 1 (El Croquis n.53, OMA/Rem Koolhaas, 1987-1993. Madrid: editorial El croquis, 1992, p. 167), 2 (Faucherau, Serge: Malévich, Barcelona: Ediciones Polígrafa, 1992, p. 143, figura 37), 3 (Aureli, Pier Vittorio: The Possibility of an absolute architecture. Cambridge: The MIT Press, 2011, p. 179); página 81, 4 (Gargiani, Roberto: Rem Koolhaas / OMA, the Construction of Maravilles. Lausanne: EPFL Press, 2008, p. 26 ), 5 (El Croquis n.53, OMA/Rem Koolhaas, 19871993. Madrid: editorial El croquis 1992, p. 169); página 82, 6 (El Croquis n.53, OMA/Rem Koolhaas, 1987-1993. Madrid: editorial El croquis 1992, p. 178), 7 (El Croquis n.53, OMA/Rem Koolhaas, 1987-1993. Madrid: editorial El croquis 1992, p. 173), 8 (El Croquis n.53, OMA/Rem Koolhaas, 1987-1993. Madrid: editorial El croquis 1992, p. 85), 9 (Aureli, Pier Vittorio: The Possibility of an absolute architecture. Cambridge: The MIT Press, 2011, p. 207); página 84, 10 (Venturi, Robert: Complexity and Contradicction in Architecture. New York: The Museum of Modern Art, 1966. p. 74), 11 ( AV Monografías 51-52, Enero-Abril 1995, p. 172), 12 (Arquitectura Viva n. 39, Noviembre-Diciembre 1994, p.43); página 86, 13 y 14 (OMA; Koolhaas, Rem; Mau, Bruce: SMLXL. Nueva York: The Monacelli Press, 1995. pp. 810-811); página 90, 1 (Courtesy of The MIT Press, from Grant Hildebrand, Designing for Industry: The Architecture of Albert Kahn. p.46), 2 (From the Collections of The Henry Ford); página 92, 3 (Nelson, George: Industrial Architecture of Albert Kahn, Inc. New York: Architectural Book Publishing Company, Inc, 1939, p.85. Foto: Hedrich-Blessing); página 93, 4 (Goldsmith, Myron: The Tall Building: the Effects of Scale, IIT, Chicago, 1953 (Tesis doctoral no publicada, revisada en. 1977 y 1986). [Versión consultada en: Goldmith, Myron y Werner Blaser (ed), Buildings and Concepts. New York: Rizzoli International Publications, 1987. pp.8-22.], p. 15), 5 (Goldsmith, M., op. cit. p. 17); página 94, 6 y página 95, 7 (Silvia Colmenares Vilata); página 96, 8 (Achilles, Rolf; Kevin Harrington and Charlotte Myhrum (ed.) Mies van der Rohe, architect as educator. Catalogue for the exhibition, 6 June through 12 July 1986 . Mies van der Rohe Centennial Project, IIT. Chicago: University of Chicago Press, 1986. pp.126 // Nelson, G. Op. cit. p. 38); página 97, 9 (Carter, Peter: Mies van der Rohe at Work. New York: Praeger, 1974. [Ed. Consultada: London: Phaidon, 1999, p. 8); página 98, 10 (Silvia Colmenares Vilata); página 99, 11 (Hvattum, Mari; Hermansen Cordua, Christian (eds.) Tracing Modernity: Manifestations of the Modern in Architecture and the City. London: Routledge, 2004. p. 126), 12 (Wesemael, Pieter van. Architecture of Instruction and Delight: A Socio-Historical Analysis of World Exhibitions As a Didactic Phenomenon (1798-1851-1970).Rotterdam: Uitgeverij 010, 2001. p. 170. (Manipulada)); página 100, 13 (Cedric Price fonds. Collection Centre Canadien d'Architecture/ Canadian Centre for Architecture, Montréal), 14 (Cedric Price fonds. Collection Centre Canadien d'Architecture/ Canadian Centre for Architecture, Montréal); página 101, 15 (Silvia Colmenares Vilata); página 106, 1, 2 y 3; página 108, 4 y 5 (Luis Palacios Labrador, 2011); página 110, 6 (Dibujo Luis Palacios Labrador, 2011. Fotografía: Strauven, Francis: Aldo Van Eyck. The Shape of Relativity. Amsterdam: Architectura \& Natura, 1998, pp. 375); página 113, 7 (Strauven, Francis: Aldo Van Eyck. The Shape of Relativity. Amsterdam: Architectura \& Natura, 1998, pp. 378), 8 (Strauven, Francis: Aldo Van Eyck. The Shape of Relativity. Amsterdam: Architectura \& Natura, 1998, p. 402); página 114, 9 (Risselada, Max; Van den Heuvel, Dirk: Team 10, 1953-81, in search of a Utopia of the present. Rotterdam: Nai Publishers, 2005, p. 117), 10 (Aldo van Eyck. The Web and the Labyrinth. En Lotus International. V.11. 1976); página 115, 11 (Sarkis, Hashim: Case: Le Corbusier's Venice Hospital and the Mat Building Revival. Munich: Prestel Verlag, 2001, p. 41); página 120, 1 (Antonio Millán Gómez, Marisol Jiménez, Julio Alan Latre y Víctor Díaz-Asensio García); página 123, 2 (Latre Cabrera, Julio Alan y Jiménez Rivera, Marisol); página 124, 3 (3.a. Busquets, Joan: El centro Histórico de Barcelona, un Pasado con futuro. Barcelona: Ajuntament de Barcelona, Foment Ciutat Vella; Universitat Politècnica de Catalunya, 2003; 3.b www.europeana.eu/portal/record/91932/CA529705B53B599FDD3C9A1ED7D5130497F5D000.html), 4 (Antonio Millán Gómez, Marisol Jiménez, Julio Alan Latre y Víctor Díaz-Asensio García); página 126, 5 (Latre Cabrera, Julio Alan y Jiménez Rivera, Marisol); página 128, 6 (llustre Municipalidad de Santiago); página 129, 7 (Archivo Visual de Santiago (www.archivovisual.cl) e llustre Municipalidad de Santiago / Santiago Centro. pp. 24-25); página 130, 8 (Lazo Mella, Felipe ; Millán Gómez, Antonio); página 131, 9 (Atlas del Proyecto de saneamiento del subsuelo de BarceIona. Lamina 3. Archivo Histórico de la Ciudad de Barcelona) 